\title{
Radiomic features of glucose metabolism enable prediction of outcome in mantle cell lymphoma
}

\author{
Marius E. Mayerhoefer ${ }^{1,2} \cdot$ Christopher C. Riedl $^{1} \cdot$ Anita Kumar $^{3} \cdot$ Peter Gibbs $^{1} \cdot$ Michael Weber $^{2} \cdot$ Ilan Tal $^{4}$. \\ Juliana Schilksy ${ }^{1} \cdot$ Heiko Schöder ${ }^{1}$
}

Received: 19 April 2019 / Accepted: 11 June 2019 / Published online: 8 July 2019

(C) The Author(s) 2019

\begin{abstract}
Purpose To determine whether $\left[{ }^{18} \mathrm{~F}\right] \mathrm{FDG}$ PET/CT-derived radiomic features alone or in combination with clinical, laboratory and biological parameters are predictive of 2-year progression-free survival (PFS) in patients with mantle cell lymphoma (MCL), and whether they enable outcome prognostication.

Methods Included in this retrospective study were 107 treatment-naive MCL patients scheduled to receive CD20 antibody-based immuno(chemo)therapy. Standardized uptake values (SUV), total lesion glycolysis, and 16 co-occurrence matrix radiomic features were extracted from metabolic tumour volumes on pretherapy $\left[{ }^{18} \mathrm{~F}\right] \mathrm{FDG}$ PET/CT scans. A multilayer perceptron neural network in combination with logistic regression analyses for feature selection was used for prediction of 2-year PFS. International prognostic indices for MCL (MIPI and MIPI-b) were calculated and combined with the radiomic data. Kaplan-Meier estimates with log-rank tests were used for PFS prognostication.

Results SUVmean (OR 1.272, $P=0.013$ ) and Entropy (heterogeneity of glucose metabolism; OR 1.131, $P=0.027$ ) were significantly predictive of 2-year PFS: median areas under the curve were 0.72 based on the two radiomic features alone, and 0.82 with the addition of clinical/laboratory/biological data. Higher SUVmean in combination with higher Entropy (SUVmean $>3.55$ and entropy $>3.5$ ), reflecting high "metabolic risk", was associated with a poorer prognosis (median PFS 20.3 vs. 39.4 months, HR 2.285, $P=0.005$ ). The best PFS prognostication was achieved using the MIPI-bm (MIPI-b and metabolic risk combined): median PFS 43.2, 38.2 and 20.3 months in the low-risk, intermediate-risk and high-risk groups respectively $(P=$ $0.005)$.

Conclusion In MCL, the $\left[{ }^{18} \mathrm{~F}\right]$ FDG PET/CT-derived radiomic features SUVmean and Entropy may improve prediction of 2-year PFS and PFS prognostication. The best results may be achieved using a combination of metabolic, clinical, laboratory and biological parameters.
\end{abstract}

Keywords Lymphoma $\cdot$ FDG $\cdot$ PET/CT $\cdot$ Prognosis

This article is part of the Topical Collection on Advanced Image Analyses (Radiomics and Artificial Intelligence)

Marius E. Mayerhoefer

mayerhom@mskcc.org

1 Department of Radiology, Molecular Imaging and Therapy Service, Memorial Sloan Kettering Cancer Center New York, 1275 York Ave, New York, NY 10065, USA

2 Department of Biomedical Imaging and Image-Guided Therapy, Medical University of Vienna, Vienna, Austria

3 Department of Medicine, Lymphoma Service, Memorial Sloan Kettering Cancer Center New York, New York, NY, USA

4 Haifa, Israel

\section{Introduction}

Mantle cell lymphoma (MCL) is a rare subtype of B cell nonHodgkin lymphoma, and can be associated with an aggressive or, less frequently, an indolent course [1]. Despite the availability of novel types of treatment, the prognosis in MCL patients is generally considered to be poor [2], with 5-year survival rates as low as $50 \%$ [1]. For estimation of prognosis, adapted versions of the International Prognostic Score (IPI) the so-called MIPI scores, which incorporate age, ECOG performance status, leucocyte count, lactic dehydrogenase levels and in some variants also the $\mathrm{Ki}-67$ proliferation index - are used in clinical practice [1]. These MIPI scores were built upon data for, and used for prediction of, 5-year survival, with 
a focus on overall survival (OS). No clinical, laboratory, or biological markers are currently established for prediction of shorter term clinical outcomes.

Pretherapy positron emission tomography/computed tomography after injection of the radiolabelled glucose analogue $2{ }^{18}{ }^{\mathrm{F}}$-fluoro-2-deoxy-D-glucose $\left(\left[{ }^{18} \mathrm{~F}\right] \mathrm{FDG}\right.$ PET/CT), which enables whole-body in vivo quantification of tumour glucose metabolism, has been shown to provide prognostic information in Hodgkin, diffuse large B cell (DLBCL), follicular and $\mathrm{T}$ cell lymphomas in a considerable number of studies, using quantitative parameters including the maximum standardized uptake value (SUVmax), total metabolic tumour volume (TMTV) and total lesion glycolysis (TLG) [3-9]. In MCL patients, only two studies have investigated the prognostic value of pretherapy SUVmax, with only one of these also including TMTV and TLG $[10,11]$. The prognostic value of quantitative measures of $\left[{ }^{18} \mathrm{~F}\right] \mathrm{FDG}$ uptake heterogeneity across the TMTV, as can be provided by advanced radiomic analyses, have not so far been investigated in MCL patients.

Radiomics is an emerging field of research that is concerned with the computer-assisted extraction of quantitative, minable data from diagnostic medical images. Radiomic features include both traditional, first-order features (such as mean and maximum grey-level values), and more sophisticated features such as those that describe different aspects of image texture, which cannot be perceived by the human eye [12]. These image textural features have the potential to allow assessment of tumour heterogeneity [13, 14], which is recognized as a prognostic determinant of survival in different types of cancer [15-17]. Indeed, several studies in different types of cancer, and using different imaging techniques, have provided data that support the prognostic potential of radiomics [18-21], especially when processed by artificial intelligencebased machine-learning algorithms.

We therefore aimed to determine (1) whether $\left[{ }^{18} \mathrm{~F}\right] \mathrm{FDG}$ PET-derived radiomic features can predict 2-year progression-free survival (PFS), alone or in combination with clinical, laboratory and biological parameters, using a machinelearning algorithm, and (2) whether the $\left[{ }^{18} \mathrm{~F}\right] \mathrm{FDG}$ PETbased radiomic signature has prognostic value in comparison to, as well as in combination with, the established MIPI scores, in MCL patients receiving CD20 antibody-based immuno(chemo)therapy as first-line systemic treatment.

\section{Materials and methods}

\section{Patients and design}

Treatment-naive patients with histologically proven MCL (as diagnosed by a reference pathologist according to the current WHO classification), who had undergone $\left[{ }^{18} \mathrm{~F}\right] \mathrm{FDG}$ PET/CT for routine pretherapy staging at a single tertiary care centre between January 2010 and June 2016, were eligible for inclusion in this Health Insurance Portability and Accountability Act (HIPAA)-compliant, retrospective study. The study was approved by the Institutional Review Board of Memorial Sloan Kettering Cancer Center; informed consent was waived. Additional inclusion criteria were: documentation of clinical follow-up and imaging follow-up (by contrastenhanced CT or $\left[{ }^{18} \mathrm{~F}\right] \mathrm{FDG}$ PET/CT) over a period of at least 2 years, or up to the date of death or progression within the 2year observation period; clinical, laboratory and biological data, including ECOG performance status, white blood cell count (WBC), lactate dehydrogenase levels (LDH) and Ki-67 proliferation index, obtained within 1 week of the pretherapy PET/CT scan; and treatment with an R-CHOP-based regimen (rituximab, cyclophosphamide, doxorubicin, vincristine and prednisone, alone or in combination with high-dose cytarabine (HiDAC) and consecutive high-dose therapy and autologous stem cell therapy (HDT/ASCT) consolidation, or radioimmunotherapy with $\left[{ }^{90} \mathrm{Y}\right]$-ibritumomab tiuxetan), or RBENDA/O-BENDA (rituximab or ofatumumab, and bendamustine), or rituximab or ofatumumab monotherapy (in patients with low tumour burden). Patients with blood glucose levels $>180 \mathrm{mg} / \mathrm{dL}$ and patients not examined with one of five prespecified PET/CT scanners (see below) were excluded.

\section{Imaging protocol}

PET/CT covering the anatomy from the mid-skull to the upper thigh was performed approximately $60 \mathrm{~min}$ after intravenous administration of $12-15 \mathrm{mCi}$ of $\left[{ }^{18} \mathrm{~F}\right] \mathrm{FDG}$. Patients fasted for at least $6 \mathrm{~h}$ prior to $\left[{ }^{18} \mathrm{~F}\right] \mathrm{FDG}$ injection. PET was performed in three-dimensional (3D) mode, with at least $3 \mathrm{~min}$ per bed position, and a voxel size of $5.5 \times 5.5 \times 3.3 \mathrm{~mm}$, using one of the following PET/CT scanners: Discovery ST, Discovery STE, Discovery 600, Discovery 690, or Discovery 710 (all manufactured by GE Healthcare, Waukesha, WI, USA). Spiral CT was performed with a tube current of $60 \mathrm{mAs}$, a tube voltage of $120-140 \mathrm{kVp}$, and a 5-mm section thickness, and was used for PET attenuation correction and anatomical correlation.

\section{Image analysis and radiomic signature}

Using the Beth Israel PET/CT viewer plugin for FIJI [22], TMTVs were semiautomatically constructed, using the previously recommended $41 \%$ SUVmax threshold (Fig. 1) [23]. When there was low $\left[{ }^{18} \mathrm{~F}\right] \mathrm{FDG}$ uptake relative to the surrounding tissues, coregistered $\mathrm{CT}$ was used to aid manual lesion delineation. Based on the TMTVs, the SUVmax, SUVmean, SUVpeak and TLG (product of TMTV and SUVmean), as well as the following 16 textural features derived from the grey-level co-occurrence matrix were 


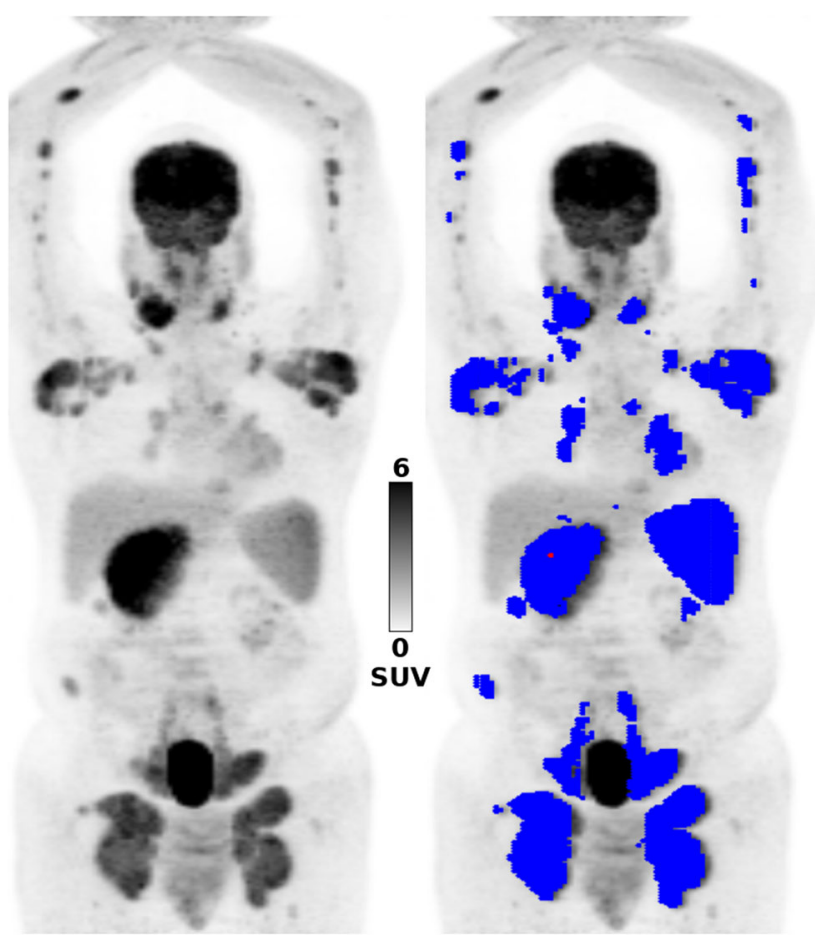

Fig. 1 A 66-year-old patient with stage IV mantle cell lymphoma: left $\left[{ }^{18} \mathrm{~F}\right] \mathrm{FDG}$ PET maximum intensity projection image; right $3 \mathrm{D}$ radiomic analysis based on the total metabolic tumour volumes (blue) constructed using the previously recommended $41 \%$ SUVmax threshold; the SUVmax (red dot) was measured in the periportal nodal bulk

calculated in 3D: Entropy, Homogeneity, Contrast, Correlation, Angular second moment, Difference entropy, Difference variance, Inverse difference moment, Sum average, Sum entropy, Sum variance, Cluster prominence, Cluster shade, Maximum probability, and two Informational measures of correlation [24]. Equations for these textural features can be accessed at https://pyradiomics.readthedocs.io/ en/latest/features.html. The 3D co-occurrence matrix was calculated with an interpixel distance of 1 , and 13 directions; a minimum of 20 pixel pairs were used for each direction. Based on this, an arithmetic mean was calculated to provide a single value for each individual feature.

Radiomic features were harmonized using the previously described ComBat method to correct for technical differences between the PET/CT data from the different scanners. ComBat harmonization was originally described for use with genomic data and was subsequently validated for normalization of PET radiomic features so as to remove the centre effect while retaining the pathophysiological information [25]. This method is applied directly to the numerical values of the radiomic features, rather than to the PET images from which the radiomic features are calculated, and consequently, it does not lead to a reduction in the quality of subsets of images. ComBat-based transformations differ between the individual radiomic features obtained from each VOI and scanner, and are applied to the measured data so that they can be pooled without the need for separate training datasets [25].

Pearson and Kendall tau-b correlation coefficients were used, as appropriate, to evaluate the relationships between the different radiomic features, and also between radiomic features and overall PFS (in months) as well as 2-year PFS status, respectively. Univariate binary logistic regression analyses were used to identify radiomic features that were significantly predictive, at $P \leq 0.05$, of 2-year PFS (i.e. 2-year PFS achieved or not). Based on radiomic features that were statistically significant in the univariate analysis, a multivariate logistic regression analysis with forward selection (based on likelihood ratio) was performed. Features that were significant in the multivariate analysis were regarded as representing the radiomic "signature" of MCL on $\left[{ }^{18} \mathrm{~F}\right] \mathrm{FDG}$ PET.

\section{Clinical data and MIPI score calculation}

ECOG performance status, WBC, LDH level, Ki-67, Ann Arbor stage and blastoid differentiation (including blastic and pleomorphic variants) of the patients with MCL were recorded. Two established variants of the MIPI score were calculated, as previously described:

- The "classic" MIPI (based on age, ECOG performance status, WBC and LDH level) with three risk categories (high, intermediate, and low risk) [26]

- The "biological" MIPI-b (which also considers the Ki-67 index) with three risk categories (high, intermediate, and low risk) [26]

Simplified versions of the MIPI scores were not obtained. PFS was obtained from the electronic medical records of the hospital information system using the oncologist's assessment and the original $\left[{ }^{18} \mathrm{~F}\right] \mathrm{FDG}$ PET/CT and CT reports. For PET/ $\mathrm{CT}$ and $\mathrm{CT}$, the Lugano response criteria for disease progression were applied [27].

\section{Machine learning for 2-year PFS prediction}

A multilayer perceptron (MLP) feed-forward artificial neural network which relies on a back-propagation learning algorithm [28] was used to determine whether the $\left[{ }^{18} \mathrm{~F}\right] \mathrm{FDG}$ $\mathrm{PET} / \mathrm{CT}$ radiomic signature can predict 2-year PFS. Because the starting point of the neural network is an initial guess at the weights of the individual radiomic features, the classification step was performed five times. The population of 107 patients was split into a training dataset and a validation dataset to which $70 \%$ and $30 \%$, respectively, were randomly assigned; i.e. randomization of patients to the training and validation datasets differed for each repetition of the classification step. A minimum of one hidden layer (activation function: hyperbolic tangent), with a minimum of three neurons per hidden 
layer, was used for the MLP neural network (output activation function: softmax). Following the purely radiomics-based analysis described above, the classification step was repeated, again five times, this time using ECOG performance status, WBC, LDH level and Ki-67 index as additional input variables, to determine whether the integration of radiomic, clinical, laboratory and biological data could improve 2-year PFS prediction. Areas under the receiver operating characteristic (ROC) curves (AUCs) as well as classification accuracies for training and validation datasets were used as the main outcome measures.

\section{Radiomic signature and MIPI for PFS prognostication}

Radiomic signature features were dichotomized using their respective ROC curve-based cut-off values. A single ordinal radiomic signature parameter reflecting the "metabolic risk" was then calculated using a "majority vote" system, with the categories "high metabolic risk" and "low metabolic risk", and, in case of an even number of radiomic signature features, an additional "intermediate metabolic risk" category (same number of features above and below their respective cut-off values). Metabolic risk, as well as MIPI and MIPI-b were tested for PFS prognostication using Kaplan-Meier estimates, and the log-rank test was used for group comparisons. To determine whether the addition of metabolic risk improved MIPI risk categories in terms of PFS prognostication, the following strategy for MIPI and MIPI-b score modification was used:
- "High metabolic risk": MIPI score +1 , unless already highest score (i.e. MIPI or MIPI-b score 3, in which case MIPI score unmodified)

- "Low metabolic risk": MIPI score -1, unless already lowest score (i.e. MIPI or MIPI-b score 1, in which case MIPI score unmodified)

- "Intermediate metabolic risk" (if applicable): MIPI score unmodified

Based on these modified MIPI and MIPI-b scores (termed "MIPI-m" and "MIPI-bm"), which included information about the metabolic risk, Kaplan-Meier estimates and logrank tests were again performed. All statistical tests were performed using SPSS 24.0 (IBM Corp., Armonk, NY, USA).

\section{Results}

A total of 107 consecutive patients ( 35 women and 72 men; mean age $64.5 \pm 10.8$ years) met the criteria for participation in the study (Fig. 2). $\left[{ }^{18} \mathrm{~F}\right] \mathrm{FDG}$ PET/CT was performed using the Discovery STE scanner in 43 patients, the Discovery 690 scanner in 41 patients, the Discovery 600 and 710 scanners in 10 patients each, and the Discovery ST scanner in 3 patients. Of the 107 patients, 58 were treated with an R-CHOP-based regimen (with additional HiDAC and HDT/ASCT in 42 patients, of whom 10 also received radioimmunotherapy), 38 with R-BENDA or O-BENDA, and 11 with rituximab or ofatumumab monotherapy. After 2 years, progression had

Fig. 2 CONSORT diagram

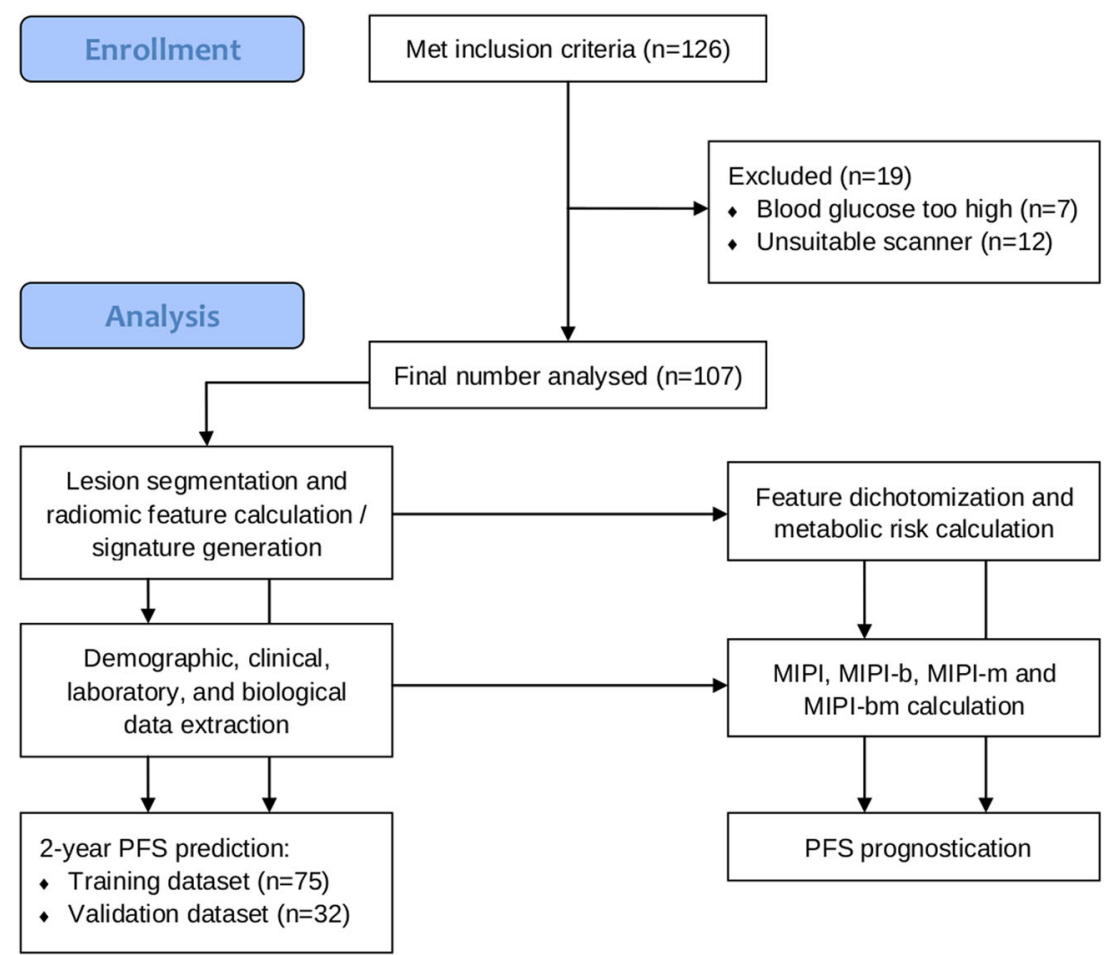


occurred in 35 of the107 patients (37.2\%), including 8 who had died. The median follow-up period was 52.6 months from registration, the median PFS was 50.4 months, and the median time to progression was 16.3 months. The patients' baseline clinical, laboratory and biological characteristics are provided in Table 1.

\section{Radiomic signature}

While radiomic features were not significantly correlated with absolute PFS (in months), 2-year PFS status was significantly correlated with SUVmean $(r=0.21, P=0.008)$, SUVpeak $(r=0.19, P=0.015)$, SUVmax $(r=0.19, P=0.018)$, Entropy $(r=0.20, P=0.012)$, Angular second moment $(r=$ $0.18, P=0.042)$ and Sum entropy $(r=0.17, P=0.033)$. SUVmean (odds ratio, OR, 1.272, 95\% confidence interval, CI, $1.037-1.560 ; P=0.021)$ and Entropy (OR 5.070, 95\% CI $1.156-22.234 ; P=0.031$ ) were the only radiomic features that were significantly predictive of 2-year PFS in the univariate analysis. Both SUVmean and Entropy, which did not show a significant correlation with each other $(r=0.17, P=0.077$ ), retained their statistical significance in the multivariate analysis $(P=0.022$ and $P=0.034$, respectively). Mean SUVmean was 3.78 (range 0.95-14.49) and mean Entropy was 3.48 (range 2.35-4.13). ROC analyses revealed an optimal SUVmean cut-off value of 3.55, and an optimal Entropy cutoff value of 3.5.
Machine learning for 2-year PFS outcome prediction

With absolute SUVmean and Entropy values as input for the neural network, AUCs for 2-year PFS prediction were $0.70-0.73$ (median 0.72); classification accuracies were $71.0-76.7 \%$ (median $74.4 \%$ ) in the training dataset and $70.6-86.8 \%$ (median $74.3 \%$ ) in the validation dataset. When, in addition to SUVmean and Entropy, ECOG performance status, WBC, LDH level and Ki-67 were added as inputs for the neural network, AUCs for 2-year PFS prediction were $0.77-0.83$ (median 0.82; Fig. 3); here, classification accuracies were $72.5-82.9 \%$ (median 79.2\%) in the training dataset, and $69.2-84.0 \%$ (median $76.7 \%$ ) in the validation dataset.

\section{Radiomic signature and MIPI for PFS prognostication}

The $\left[{ }^{18} \mathrm{~F}\right]$ FDG PET radiomic signature, consisting of dichotomized SUVmax and Entropy, was used to construct a two-category and a three-category prognostic model of metabolic risk for progression. In the two-category model, patients with high metabolic risk (SUVmean $>3.55$ and Entropy $>3.5$ ) had a median PFS of 20.3 months, with a 2-year PFS of $40.7 \%$ (11/27 patients), whereas patients with low metabolic risk (SUVmean $\leq 3.55$ and/ or Entropy $\leq 3.5$ ) had a median PFS of 39.4 months, with a 2-year PFS of $76.3 \%$ (61/80 patients). PFS differed
Table 1 Baseline demographic, clinical, laboratory and biological data of 107 MCL patients, and the results of binary logistic regression analyses for continuous and categorical data

\begin{tabular}{llll}
\hline \multirow{2}{*}{ Characteristic } & Frequency & \multicolumn{2}{l}{ Univariate analysis for 2-year PFS } \\
\cline { 4 - 4 } & & OR $(95 \%$ CI $)$ & $P$ value \\
\hline Age & - & $1.000(0.961-1.041)$ & 1.0 \\
$\geq 65$ years & $51(47.7 \%)$ & $1.251(0.557-2.810)$ & 0.59 \\
Ann Arbor stage & - & - & - \\
I & $5(4.7 \%)$ & 1 & - \\
II & $13(12.1 \%)$ & $1.286(0.158-10.450)$ & 0.81 \\
II & $23(21.5 \%)$ & $0.417(0.054-3.221)$ & 0.40 \\
IV & $66(61.7 \%)$ & $0.750(0.117-4.822)$ & 0.76 \\
Blastoid differentiation & $20(18.7 \%)$ & $0.857(0.298-2.462)$ & 0.78 \\
Blastic & $18(16.8 \%)$ & - & - \\
Pleormorphic & $2(1.9 \%)$ & - & - \\
WBC & - & $1.017(0.982-1.053)$ & 0.35 \\
Elevated & $18(16.8 \%)$ & $1.837(0.653-5.165)$ & 0.25 \\
Ki-67 index & - & $1.007(0.991-1.022)$ & 0.39 \\
$\geq 30 \%$ & $56(52.3 \%)$ & $0.890(0.396-2.001)$ & 0.78 \\
LDH level & - & $1.004(0.999-1.009)$ & 0.096 \\
Elevated & $29(27.1 \%)$ & $1.116(0.453-2.748)$ & 0.81 \\
ECOG performance status & - & - & - \\
$\geq 2$ & $7(6.5 \%)$ & $1.594(0.337-7.545)$ & 0.56 \\
\hline PR & & &
\end{tabular}

$O R$ odds radio, $C I$ confidence interval 

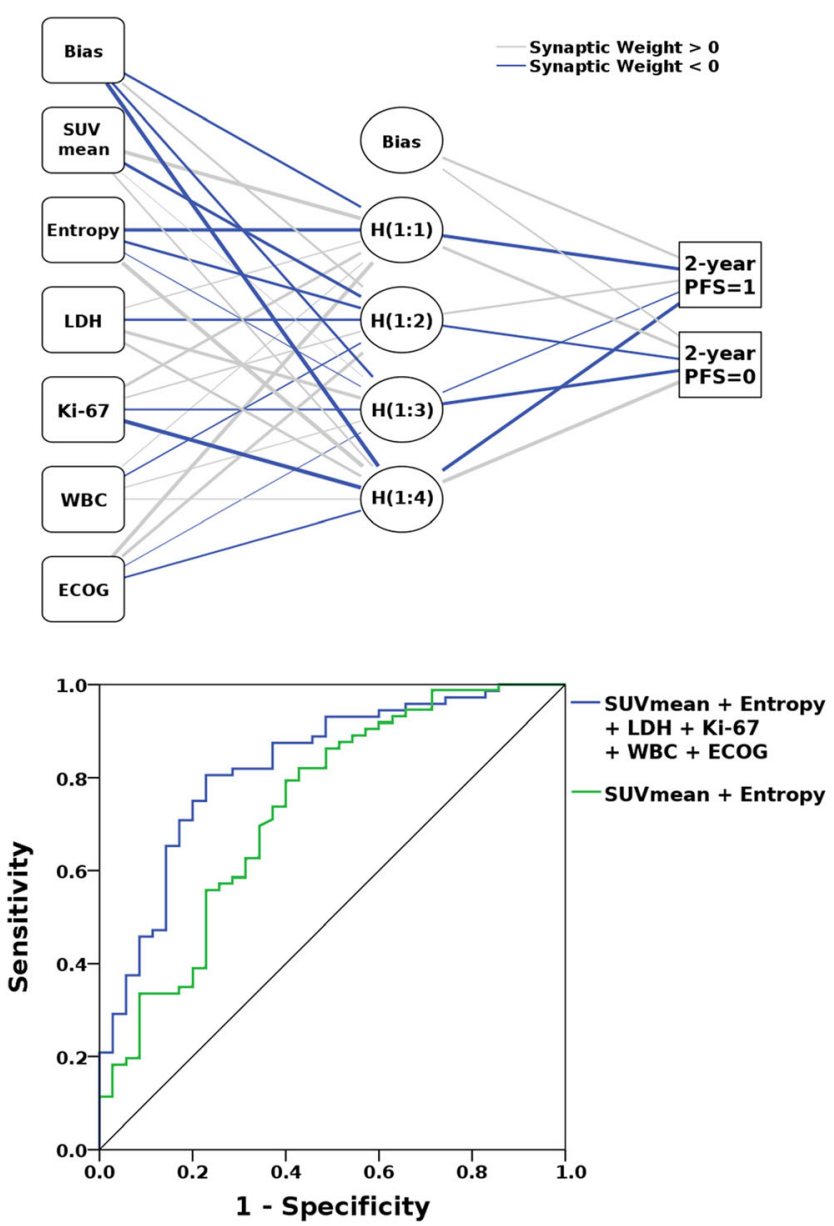

Fig. 3 Results of the multi-layer perceptron (MLP) neural network-based prediction of 2-year PFS. With a single "hidden" layer with four neurons (top $\mathrm{H}(1: 1)$ to $\mathrm{H}(1: 4)$ ), and SUVmean, Entropy, lactate dehydrogenase level (LDH), white blood count (WBC), Ki-67 index and ECOG performance status as inputs, the receiver operating characteristic (ROC) curve (bottom) yielded an area under the curve (AUC) of 0.83, whereas the use of just the two radiomic features (SUVmean and Entropy) as input for the neural network yielded an AUC of 0.73

significantly between the two groups $(P=0.005$; Table 2, Fig. 4). In the three-category model, patients with high metabolic risk (SUVmean $>3.55$ and Entropy $>3.5$ ) had a median PFS of 20.3 months, with a 2-year PFS of $40.7 \%$ (11/27 patients), patients with intermediate metabolic risk (SUVmean $\leq 3.55$ or Entropy $\leq 3.5$ ) had a median PFS of 40.3 months, with a 2-year PFS of 71.4\% (35/49 patients), and patients with low metabolic risk (SUVmean $\leq 3.55$ and Entropy $\leq 3.5$ ) had a median PFS of 38.2 months, with a 2-year PFS of $83.9 \%$ (26/ 31 patients). PFS differed significantly among the three groups ( $P=0.017$; Table 2, Fig. 4$)$.

Both metabolic risk scores (i.e. with two and three risk categories) were superior to MIPI and MIPI-b (Table 2, Fig. 4). However, the best PFS prognostication with three risk categories was achieved with MIPI-bm $(P=0.005)$, i.e. the MIPI-b modified using the three metabolic risk categories (Table 2, Fig. 4).

\section{Discussion}

Our study identified two main image-based predictors of 2year PFS in MCL patients receiving CD20 antibody-based immuno(chemo)therapy: the average glucose metabolism across the entire MTV, as reflected by the SUVmean on pretherapy $\left[{ }^{18} \mathrm{~F}\right] \mathrm{FDG}$ PET/CT scans, and the heterogeneity of glucose metabolism within this MTV, as reflected by Entropy. The combination of the two radiomic features - i.e. the $\left[{ }^{18} \mathrm{~F}\right]$ FDG PET radiomic signature - may also be useful for outcome prediction: higher SUVmean and higher Entropy appear to be associated with a shorter PFS (Fig. 4).

The three "metabolic risk" categories based on the radiomic signature were superior to the MIPI risk categories in terms of PFS prognostication, regardless of whether or not the $\mathrm{Ki}-67$ proliferation index was considered in the calculation of the MIPI (Table 2, Fig. 4). The performance of the MIPI scores is not necessarily surprising, as OS, and not PFS, was the primary endpoint used in the development of MIPI and MIPI-b [26]. Notably, both MIPI and MIPI-b scores were considerably improved through combination with the $\left[{ }^{18} \mathrm{~F}\right]$ FDG PET-based metabolic risk. In particular, unlike the MIPI-b, the MIPI-bm not only emerged as a statistically significant predictor of PFS, but was superior to metabolic risk alone (Table 2, Fig. 4), indicating that the best results may be achieved when clinical, laboratory and biological, as well as metabolic information, are integrated in a single model. This is also supported by the results of our machine-learning experiment, in which the addition of ECOG performance status, WBC, LDH level and Ki-67 index to the radiomic features clearly improved 2-year PFS prediction (Fig. 3). Since $\left[{ }^{18} \mathrm{~F}\right] \mathrm{FDG} \mathrm{PET} / \mathrm{CT}$ is currently recommended for staging and treatment response assessment in patients with MCL by the International Conference on Malignant Lymphoma (ICML) [27] - i.e. it is considered a standard procedure in these patients - information on the metabolic risk represents routine data, which may facilitate its integration into risk assessment in clinical practice.

The prognostic value of pretherapy quantitative $\left[{ }^{18} \mathrm{~F}\right] \mathrm{FDG}$ PET/CT in treatment-naive MCL patients has, to our knowledge, only been investigated in two prior studies. In a series of 81 patients, Karam et al. used the SUVmax to identify groups of MCL patients at risk of shorter survival [10]. The design of their study differed from ours in several ways. First, the SUVmax, which provides information about the single voxel with the highest glucose metabolism within the tumour volume, was the only $\left[{ }^{18} \mathrm{~F}\right]$ FDG PET-based parameter evaluated, whereas radiomic analysis as performed in our study captures multiple facets of glucose metabolism across the entire metabolic lymphoma volume. Second, patients in the study by Karam et al. were chiefly treated with single-agent or combination chemotherapy (e.g. chlorambucil or CHOP), which does not reflect the present-day therapeutic state-of-the-art 
Table 2 Descriptive data and results of log-rank tests for MIPIs, with and without modification due to "metabolic risk" on $\left[{ }^{18} \mathrm{~F}\right] \mathrm{FDG} \mathrm{PET} / \mathrm{CT}$, for $107 \mathrm{MCL}$ patients

\begin{tabular}{lllll}
\hline Characteristic & Frequency & $\begin{array}{l}\text { Median PFS } \\
\text { (months) }\end{array}$ & $\begin{array}{l}\text { Hazard radio } \\
(95 \% \text { CI })\end{array}$ & $P$ value \\
\hline Metabolic risk $^{\mathrm{a}}-$ two category model & - & - & - & 0.005 \\
Low risk & $80(74.8 \%)$ & 20.3 & 1 & - \\
High risk & $27(25.2 \%)$ & 39.4 & $2.285(1.264-4.131)$ & 0.005 \\
Metabolic risk ${ }^{\mathrm{a}}-$ three category model & - & - & - & 0.017 \\
Low risk & $31(29.0 \%)$ & 38.2 & 1 & - \\
Intermediate risk & $49(45.8 \%)$ & 40.3 & $1.225(0.590-2.543)$ & 0.59 \\
High risk & $27(25.2 \%)$ & 20.3 & $2.597(1.212-5.564)$ & 0.14 \\
MIPI & - & - & - & 0.27 \\
Low risk & $30(28.0 \%)$ & 41.7 & 1 & - \\
Intermediate risk & $45(42.1 \%)$ & 38.1 & $0.866(0.424-1.771)$ & 0.69 \\
High risk & $32(29.9 \%)$ & 27.7 & $1.463(0.715-2.992)$ & 0.30 \\
MIPI-b & - & - & - & 0.37 \\
Low risk & $15(14.0 \%)$ & 43.8 & 1 & - \\
Intermediate risk & $36(33.6 \%)$ & 35.7 & $1.441(0.523-3.969)$ & 0.48 \\
High risk & $56(52.3 \%)$ & 32.0 & $1.872(0.724-4.843)$ & 0.20 \\
MIPI-m & - & - & - & 0.14 \\
Low risk & $35(32.7 \%)$ & 41.7 & 1 & - \\
Intermediate risk & $41(38.3 \%)$ & 37.6 & $1.344(0.657-2.750)$ & 0.42 \\
High risk & $31(29.0 \%)$ & 26.6 & $2.013(0.984-4.120)$ & 0.055 \\
MIPI-bm & - & - & - & 0.005 \\
Low risk & $20(18.7 \%)$ & 43.2 & 1 & - \\
Intermediate risk & $58(54.2 \%)$ & 38.2 & $2.675(0.935-7.653)$ & 0.066 \\
High risk & $29(27.1 \%)$ & 20.3 & $4.884(1.647-14.607)$ & 0.004 \\
\hline & & & & \\
\hline
\end{tabular}

$H R$ hazard radio, relative to low risk group, CIconfidence interval

${ }^{a}$ Based on the radiomic signature (combination of dichotomized SUVmean and Entropy)

${ }^{\mathrm{b}}$ Modified according to metabolic risk for first-line systemic treatment in MCL [29]. Third, three different PET scanners were used, but no correction was performed for the technical differences. Finally, while Karam et al. were able to successfully identify two MCL risk categories using an SUVmax cut-off value of 5, a further subdivision of MCLs with SUV >5 failed; no combination with MIPI scores or individual clinical/laboratory/biological data was attempted. In the second, very recent study, Albano et al. retrospectively evaluated three quantitative $\left[{ }^{18} \mathrm{~F}\right] \mathrm{FDG} \mathrm{PET} / \mathrm{CT}$ parameters - baseline SUVmax, TMTV and TLG - for outcome prediction in $87 \mathrm{MCL}$ patients, using two different PET/ CT scanners, also without applying correction for possible technical differences. These authors found that TMTV and TLG, but not SUVmax, were significantly associated with PFS using two risk categories. Contrary to our own study, neither SUVmean nor radiomic textural features reflecting the heterogeneity of glucose metabolism across the entire tumour, were included in their analysis, and no comparison or combination with MIPI scores was performed [11].

Entropy, a radiomic textural feature derived from the cooccurrence matrix, describes the degree of randomness, or disorder, in the distribution of image voxel grey-level values.
Moon et al. recently demonstrated that $\left[{ }^{18} \mathrm{~F}\right] \mathrm{FDG}$ PET/CTderived Entropy is correlated with the genetic heterogeneity index in lung cancer [13], whereas Choi et al. found that dualenergy CT-derived radiomic features, including Entropy, are also strongly correlated with the pathological heterogeneity index in lung cancer [14]. Entropy extracted from $\left[{ }^{18} \mathrm{~F}\right] \mathrm{FDG}$ PET/CT has recently been used for PFS prediction in patients with high-risk squamous cell carcinoma of the oropharynx after chemoradiation [30], and in patients with lung cancer after EGFR tyrosine kinase inhibitor treatment [31], and has also been found to be associated with failure to respond to third-line systemic treatment in metastatic colorectal cancer [32]. In lymphoma, however, pretherapy $\left[{ }^{18} \mathrm{~F}\right]$ FDG PET/CTbased Entropy has so far been evaluated for prediction of interim response (i.e. the outcome after two therapy cycles) in paediatric Hodgkin lymphoma [33], and for prediction of disease-free survival and OS in aggressive B cell nonHodgkin lymphoma (predominantly DLBCL, with a followup period of 3-54 months) [34], but did not emerge as a statistically significant marker in either study. Our study is therefore the first to show the value of Entropy on $\left[{ }^{18} \mathrm{~F}\right] \mathrm{FDG}$ $\mathrm{PET} / \mathrm{CT}$ for outcome prediction in patients with a distinct 
Fig. 4 Kaplan-Meier estimates and log-rank tests show that the $\left[{ }^{18} \mathrm{~F}\right]$ FDG PET/CT radiomic signature (combination of SUVmean and Entropy), which reflects "metabolic risk", enables PFS prognostication. MIPI and MIPI$\mathrm{b}$ are clearly improved by combining with metabolic risk, as assessed on $\left[{ }^{18} \mathrm{~F}\right] \mathrm{FDG}$ PET/CT. The best results are achieved with MIPI-bm (i.e. combination of MIPI-b and metabolic risk)
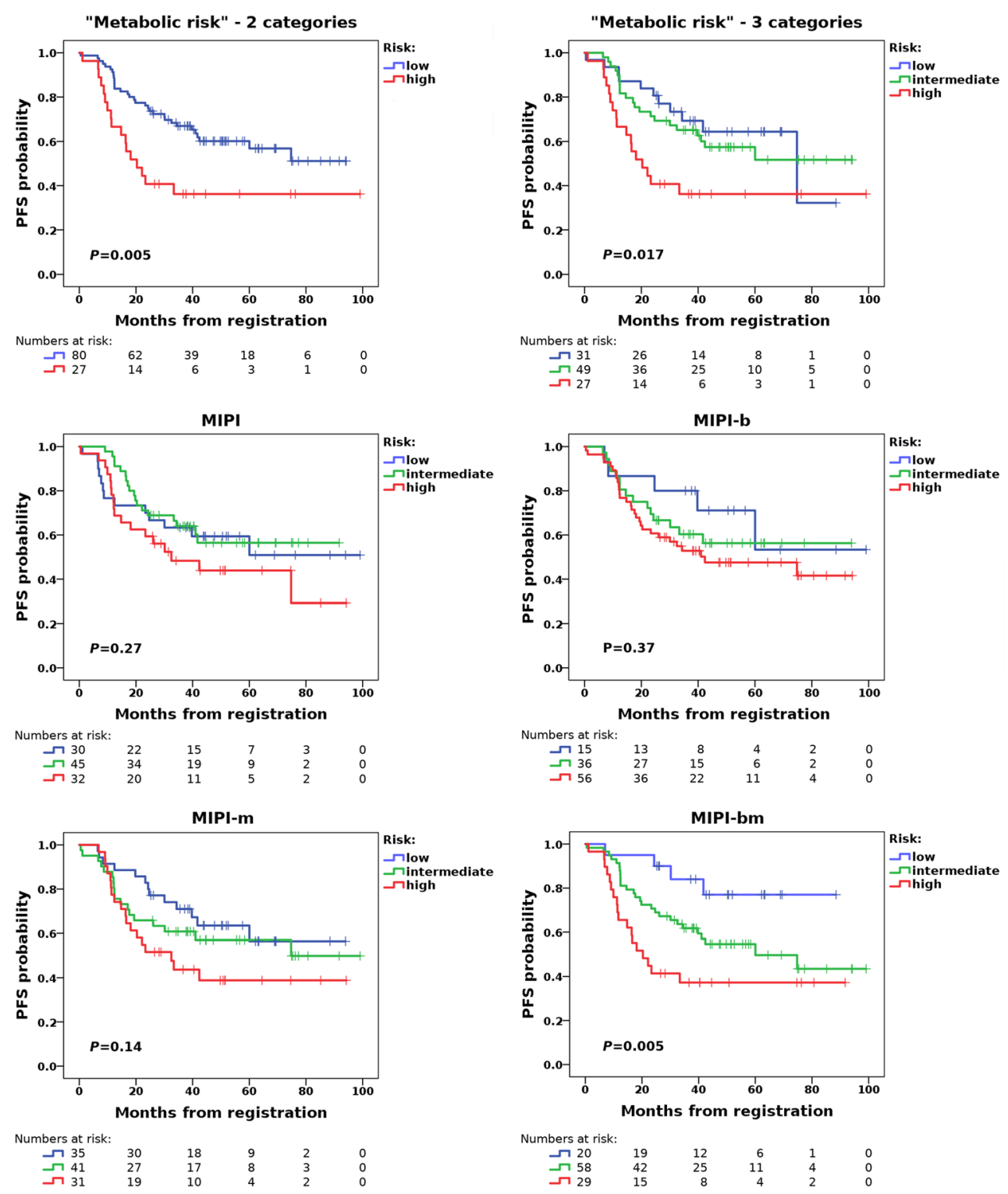

histological lymphoma subtype, a finding that could be due to the fact that MCL is a "genomically unstable" tumour that may be associated with marked (sub)clonal heterogeneity, and also with heterogeneity between different topographic sites in the same patient, and with modulation of the initial mutational profile during disease progression [35].

We used PFS instead of OS as the clinical endpoint, which is contrary to many lymphoma studies investigating prognostic features. This strategy was chosen because MCL is a less common lymphoma subtype, and hence smaller patient populations and shorter follow-up periods may have to be used to obtain a sufficiently large number of cases with the event of interest. While OS is the established outcome, PFS has been recognized by the Food and Drug Administration as a valid surrogate endpoint in MCL and other haematological malignancies [36]. In a recent analysis of multiple randomized trials in DLBCL, including a total of 7,507 patients, PFS was significantly correlated with OS, supporting its use as a surrogate marker (https:// www.fda.gov/Drugs/DevelopmentApprovalProcess/ DevelopmentResources/ucm613636.htm).

Our study had some limitations. The most obvious limitations were the retrospective design and the modest cohort size. However, this was a hypothesis-generating study, as $\left[{ }^{18} \mathrm{~F}\right] \mathrm{FDG}$ PET-derived radiomic features have not been previously evaluated for outcome prediction and prognostication in MCL patients. Furthermore, with a sample size of 107 patients, this is the largest study on this topic at present. Our approach involving the combination of radiomic features and MIPI scores - i.e. using the three categories of metabolic risk to modify MIPI scores was exploratory. However, MIPI scores were clearly improved by applying this strategy, and in addition, the combination of the (continuous) radiomic feature values and the clinical, laboratory 
and biological parameters (which were also used for calculation of the MIPI scores), using a machine-learning algorithm, showed the same trend: best results were achieved when all parameters were integrated into a single model. We used an MLP neural network for outcome prediction, which is a universal function approximator with the ability to model any type of regression or classification problem [37]. While MLP networks are well established in the machine-learning community as powerful prediction algorithms [38], it is possible that even more advanced, deep machine-learning techniques such as convolutional neural networks $(\mathrm{CNN})$, with their larger numbers of hidden layers and their interconnection between neurons within the same layer, may have performed even better. However, CNNs are mainly intended for use with large datasets ("big data"). In a patient population such as our own, however, their complexity would have increased the probability of "overfitting", i.e. loss of generalizability of the model, in our case for PFS prediction [39].

In conclusion, an $\left[{ }^{18} \mathrm{~F}\right] \mathrm{FDG}$ PET radiomic signature comprising SUVmean and Entropy has prognostic value in MCL and may be useful for predicting early tumour progression. This metabolic risk reflected by radiomic features can be integrated into MIPI scores and may possibly improve risk stratification in MCL. Further studies are warranted to validate these findings in external cohorts.

Funding Information Open access funding provided by Medical University of Vienna.

\section{Compliance with ethical standards}

Conflicts of interest M.E.M. has received speaker honoraria and research support from Siemens Healthineers, and speaker honoraria from Bristol-Myers Squibb. H.S. has received honoraria for consultancy from Aileron Therapeutics. All other authors declare no potential conflicts of interest.

Ethical approval All procedures performed in studies involving human participants were in accordance with the ethical standards of the institutional research committee (IRB) and with the principles of the 1964 Declaration of Helsinki and its later amendments or comparable ethical standards.

Informed consent Informed consent was waived by the IRB due to the retrospective design.

Open Access This article is distributed under the terms of the Creative Commons Attribution 4.0 International License (http:// creativecommons.org/licenses/by/4.0/), which permits unrestricted use, distribution, and reproduction in any medium, provided you give appropriate credit to the original author(s) and the source, provide a link to the Creative Commons license, and indicate if changes were made.

\section{References}

1. Dreyling M, Thieblemont C, Gallamini A, Arcaini L, Campo E, Hermine O, et al. ESMO consensus conferences: guidelines on malignant lymphoma. Part 2: marginal zone lymphoma, mantle cell lymphoma, peripheral T-cell lymphoma. Ann Oncol. 2013;24:857-77.

2. Hoster E, Dreyling M, Klapper W, Gisselbrecht C, van Hoof A, Kluin-Nelemans $\mathrm{HC}$, et al. A new prognostic index (MIPI) for patients with advanced-stage mantle cell lymphoma. Blood. 2008; 111:558-65.

3. Mettler J, Müller H, Voltin CA, Baues C, Klaeser B, Moccia A, et al. Metabolic tumour volume for response prediction in advanced-stage Hodgkin lymphoma. J Nucl Med. 2019;60:20711. https://doi.org/10.2967/jnumed.118.210047.

4. Meignan M, Cottereau AS, Versari A, Chartier L, Dupuis J, Boussetta S, et al. Baseline metabolic tumor volume predicts outcome in high-tumor-burden follicular lymphoma: a pooled analysis of three multicenter studies. J Clin Oncol. 2016;34:3618-26.

5. Mikhaeel NG, Smith D, Dunn JT, Phillips M, Møller H, Fields PA, et al. Combination of baseline metabolic tumour volume and early response on PET/CT improves progression-free survival prediction in DLBCL. Eur J Nucl Med Mol Imaging. 2016;43:1209-19.

6. Ceriani L, Martelli M, Zinzani PL, Ferreri AJ, Botto B, Stelitano C, et al. Utility of baseline 18FDG-PET/CT functional parameters in defining prognosis of primary mediastinal (thymic) large B-cell lymphoma. Blood. 2015;126:950-6.

7. Cottereau AS, Lanic H, Mareschal S, Meignan M, Vera P, Tilly H, et al. Molecular profile and FDG-PET/CT total metabolic tumor volume improve risk classification at diagnosis for patients with diffuse large B-cell lymphoma. Clin Cancer Res. 2016;22:3801-9.

8. Becker S, Vermeulin T, Cottereau AS, Boissel N, Vera P, Lepretre S. Predictive value of (18)F-FDG PET/CT in adults with T-cell lymphoblastic lymphoma: post hoc analysis of results from the GRAALL-LYSA LLO3 trial. Eur J Nucl Med Mol Imaging. 2017;44:2034-41.

9. Cottereau AS, El-Galaly TC, Becker S, Broussais F, Petersen LJ, Bonnet $\mathrm{C}$, et al. Predictive value of PET response combined with baseline metabolic tumor volume in peripheral T-cell lymphoma patients. J Nucl Med. 2018;59:589-95.

10. Karam M, Ata A, Irish K, Feustel PJ, Mottaghy FM, Stroobants SG, et al. FDG positron emission tomography/computed tomography scan may identify mantle cell lymphoma patients with unusually favorable outcome. Nucl Med Commun. 2009;30:770-8.

11. Albano D, Bosio G, Bianchetti N, Pagani C, Re A, Tucci A, et al. Prognostic role of baseline 18F-FDG PET/CT metabolic parameters in mantle cell lymphoma. Ann Nucl Med. 2019. https://doi.org/10. 1007/s12149-019-01354-9.

12. Yip SS, Aerts HJ. Applications and limitations of radiomics. Phys Med Biol. 2016;61:R150-66.

13. Moon SH, Kim J, Joung JG, Cha H, Park WY, Ahn JS, et al. Correlations between metabolic texture features, genetic heterogeneity, and mutation burden in patients with lung cancer. Eur J Nucl Med Mol Imaging. 2019;46:446-54.

14. Choi ER, Lee HY, Jeong JY, Choi YL, Kim J, Bae J, et al. Quantitative image variables reflect the intratumoral pathologic heterogeneity of lung adenocarcinoma. Oncotarget. 2016;7: 67302-13.

15. Burrell RA, McGranahan N, Bartek J, Swanton C. The causes and consequences of genetic heterogeneity in cancer evolution. Nature. 2013;501:338-45.

16. Morris LG, Riaz N, Desrichard A, Șenbabaoğlu Y, Hakimi AA, Makarov V, et al. Pan-cancer analysis of intratumor heterogeneity as a prognostic determinant of survival. Oncotarget. 2016;7:1005163.

17. Liu J, Dang H, Wang XW. The significance of intertumor and intratumor heterogeneity in liver cancer. Exp Mol Med. 2018;50: e416.

18. Sun R, Limkin EJ, Vakalopoulou M, Dercle L, Champiat S, Han $\mathrm{SR}$, et al. A radiomics approach to assess tumour-infiltrating CD8 cells and response to anti-PD-1 or anti-PD-L1 immunotherapy: an 
imaging biomarker, retrospective multicohort study. Lancet Oncol. 2018;1:1180-91.

19. Park H, Lim Y, Ko ES, Cho HH, Lee JE, Han BK, et al. Radiomics signature on magnetic resonance imaging: association with diseasefree survival in patients with invasive breast cancer. Clin Cancer Res. 2018;24:4705-14.

20. Arshad MA, Thornton A, Lu H, Tam H, Wallitt K, Rodgers N, et al. Discovery of pre-therapy 2-deoxy-2-(18)F-fluoro-D-glucose positron emission tomography-based radiomics classifiers of survival outcome in non-small-cell lung cancer patients. Eur J Nucl Med Mol Imaging. 2019;46:455-66.

21. Kim S, Shin J, Kim DY, Choi GH, Kim MJ, Choi JY. Radiomics on gadoxetic acid-enhanced magnetic resonance imaging for prediction of postoperative early and late recurrence of single hepatocellular carcinoma. Clin Cancer Res. 2019. https://doi.org/10.1158/ 1078-0432.CCR-18-2861.

22. Kanoun S, Tal I, Berriolo-Riedinger A, Rossi C, Riedinger JM, Vrigneaud JM, et al. Influence of software tool and methodological aspects of total metabolic tumor volume calculation on baseline [18F]FDG PET to predict survival in Hodgkin lymphoma. PLoS One. 2015;10:e0140830.

23. Boellaard R, O'Doherty MJ, Weber WA, Mottaghy FM, Lonsdale MN, Stroobants SG, et al. FDG PET and PET/CT: EANM procedure guidelines for tumour PET imaging: version 1.0. Eur J Nucl Med Mol Imaging. 2010;37:181-200.

24. Haralick R, Shanmugan K, Dinstein I. Textural features for image classification. IEEE Trans Syst Man Cybernet. 1973;3:610-21.

25. Orlhac F, Boughdad S, Philippe C, Stalla-Bourdillon H, Nioche C, Champion L, et al. A postreconstruction harmonization method for multicenter radiomic studies in PET. J Nucl Med. 2018;59:1321-8.

26. Moskowitz AJ, Schöder H, Gavane S, Thoren KL, Fleisher M, Yahalom J, et al. Prognostic significance of baseline metabolic tumor volume in relapsed and refractory Hodgkin lymphoma. Blood. 2017; 130:2196-203.

27. Cheson BD, Fisher RI, Barrington SF, Cavalli F, Schwartz LH, Zucca E, et al. Recommendations for initial evaluation, staging, and response assessment of Hodgkin and non-Hodgkin lymphoma: the Lugano classification. J Clin Oncol. 2014;32:3059-68.

28. LeCun Y, Bengio Y, Hinton G. Deep learning. Nature. 2015;521: 436-44.

29. Maddocks K. Update on mantle cell lymphoma. Blood. 2018;132: 1647-56.

30. Cheng NM, Fang YD, Tsan DL, Lee LY, Chang JT, Wang HM, et al. Heterogeneity and irregularity of pretreatment (18)F- fluorodeoxyglucose positron emission tomography improved prognostic stratification of p16-negative high-risk squamous cell carcinoma of the oropharynx. Oral Oncol. 2018;78:156-62.

31. Park S, Ha S, Lee SH, Paeng JC, Keam B, Kim TM, et al. Intratumoral heterogeneity characterized by pretreatment PET in non-small cell lung cancer patients predicts progression-free survival on EGFR tyrosine kinase inhibitor. PLoS One. 2018;13: e0189766.

32. van Helden EJ, Vacher YJL, van Wieringen WN, van Velden FHP, Verheul HMW, Hoekstra OS, et al. Radiomics analysis of pretreatment [(18)F]FDG PET/CT for patients with metastatic colorectal cancer undergoing palliative systemic treatment. Eur J Nucl Med Mol Imaging. 2018;45:2307-17.

33. Rogasch JMM, Hundsdoerfer P, Hofheinz F, Wedel F, Schatka I, Amthauer H, et al. Pretherapeutic FDG-PET total metabolic tumor volume predicts response to induction therapy in pediatric Hodgkin's lymphoma. BMC Cancer. 2018;18:521.

34. Parvez A, Tau N, Hussey D, Maganti M, Metser U. (18)F-FDG $\mathrm{PET} / \mathrm{CT}$ metabolic tumor parameters and radiomics features in aggressive non-Hodgkin's lymphoma as predictors of treatment outcome and survival. Ann Nucl Med. 2018;32:410-6.

35. Beà $\mathrm{S}$, Valdés-Mas R, Navarro A, Salaverria I, Martín-Garcia D, Jares P, et al. Landscape of somatic mutations and clonal evolution in mantle cell lymphoma. Proc Natl Acad Sci U S A. 2013;110: $18250-5$.

36. Shi Q, Schmitz N, Ou FS, Dixon JG, Cunningham D, Pfreundschuh $\mathrm{M}$, et al. Progression-free survival as a surrogate end point for overall survival in first-line diffuse large B-cell lymphoma: an individual patient-level analysis of multiple randomized trials (SEAL). J Clin Oncol. 2018;36:2593-602.

37. Mocanu DC, Mocanu E, Stone P, Nguyen PH, Gibescu M, Liotta A. Scalable training of artificial neural networks with adaptive sparse connectivity inspired by network science. Nat Commun. 2018;9:2383.

38. Ditzler G, Polikar R, Rosen G. Multi-layer and recursive neural networks for metagenomic classification. IEEE Trans Nanobiosci. 2015;14:608-16.

39. Yamashita R, Nishio M, Do RKG, Togashi K. Convolutional neural networks: an overview and application in radiology. Insights Imaging. 2018;9:611-29.

Publisher's note Springer Nature remains neutral with regard to jurisdictional claims in published maps and institutional affiliations. 